\title{
Plant syndrome recognition by Gigapixel Image using Convolutional Neural Network
}

\author{
C.Saravanakumar ${ }^{1}$, P.Gururama Senthilvel' ${ }^{2}$, D.R. Thirupurasundari ${ }^{3}$, P.N. Periyasamy ${ }^{4}$ \\ and K.Vijayakumar ${ }^{5}$ \\ \{mailofcsk@gmail.com¹, gurupandian@gmail.com², tpsdr@yahoo.com³,pnpsamy80@gmail.com ${ }^{4}$, \\ mkvijay@msn.com $\left.{ }^{5}\right\}$

\begin{abstract}
${ }^{1}$ Associate Professor, Department of Information Technology, St.Joseph's Institute of Technology, OMR, Chennai - 600119, India Meenakshi College of Engineering, Chennai - 600078, India

${ }^{3}$ Assistant Professor, Department of Computer Science and Engineering, Meenakshi College of Engineering, Chennai - 600078, India

${ }^{4}$ Assistant Professor, Department of Information Technology, Meenakshi College of Engineering, Chennai - 600078, India

${ }^{5}$ Associate Professor, Computer Science and Engineering, St.Joseph's Institute of Technology, OMR, Chennai - 600119, India
\end{abstract} \\ ${ }^{2}$ Associate Professor, Department of Computer Science and Engineering,
}

\begin{abstract}
The plants play a vital role in our day-to-day life. It is important to monitor the health of the plants. Generally, plant diseases are identified using image processing techniques. In those techniques, the input images of plants are of an only megapixel size. In this method image processing of plants is done using a gigapixel input image that covers the entire area of the crop. To process this huge gigapixel image a method called Neural Image Compression(NIC) is used. The identification of the plant diseases is done using Convolutional Neural Networks(CNN) from the neutrally compressed gigapixel image. $\mathrm{CNN}$ is trained using a probability estimation algorithm to identify the affected portion of the plant crop.
\end{abstract}

\begin{abstract}
Keywords: Gigapixel Image, Neural Image Compression(NIC), plant diseases, Convolutional Neural Networks $(\mathrm{CNN})$, Probability estimator algorithm, Image Processing
\end{abstract}

\section{Introduction}

The health of the plants determines the nutrient content of the plant. Hence it is quite important to recognize if the plant is affected by any disease. In the normal methods of image processing, only megapixel sized images of plants are given as inputs. It covers only the selected region of the plant crops and prevents us from monitoring the entire hectare of the crops by ignoring it since they are not visible. Even if there is a small portion of a plant affected in the entire hectare if it is ignored then there are chances that it can spread to the entire crop. This issue can be overcome if the input image of the plant crop is given in terms of gigapixel. It gives the entire hectare view of the plants and identifies the disease in the affected region. To process the huge gigapixel image, the corresponding image is first converted into a neutrally compressed image. A Convolutional Neural Network(CNN) is used 
to identify the type of disease found in the plant. CNN is trained using a probability estimator algorithm. To overcome the challenge of spreading diseases from a small portion to the entire hectare of the crop this gigapixel method of image processing is proposed.The Neural Image Compression(NIC) method is taken from[1] which uses a histopathology dataset to identify the presence of cancer in human cells. In that approach[1] the Convolutional Neural Networks(CNN) were trained using Variational Auto Encoder(VAE) and Bidirectional Generative Adversarial Network(BIGAN). But these methods of training faced challenges in learning and they de-limited the mentalistic lesions found. Hence they were not suitable for any other applications other than histopathology. This challenge is overcome by the probabilistic estimator algorithm which is quite general to use not only for histopathology but also for the plant diseases. Much other analysis on the plants such as ARIGAN (Synthetic Arabidopsis Plants using Generative Adversarial Network)[2], Leaf Segmentation and Tracking Using Probabilistic Parametric Active Contours[3], TRiP (Tracking Rhythms in Plants)an automated leaf movement analysis program for circadian period estimation [4], Multi-modality imagery database for plant phenotyping were also proposed. Each of these different methods had different research perspectives and applications on the plants. Their perspectives included estimating the leaf growth[2], segmentation of the leaves[3], tracking the noise of the plants [4], determining the genetics, and the hybrid variety of the plants[5] and an imagery database named MSU-PID . All these approaches had different fields of plant study but none of them used the gigapixel image approach which is essential and important in plants.

Section II contains the related works of the proposed system, section III discusses about the proposed methodology, section IV shows the experimental results, section V concludes the work and discusses some of the future directions.

\section{Related Works}

The system [2] included a flexible framework that had two phases: plant growth setting and an imaging system. Canon SD870 cameras were used to capture the leaf images. It was a megapixel image. A image analysis method with time was developed. It monitor leaf growth under the seasonal condition with variation. There were also has various fields for measuring the growth traits namely rosette, circular, and leaf fit which were determined using a normalized manner. During the process of the image analysis pipeline, the color filtering is done using the linear combinations of the pixel intensities from the RGB channels. The growth of the leaf in a crop is estimated using this method. The image analysis pipeline is shown in the figure 1 .

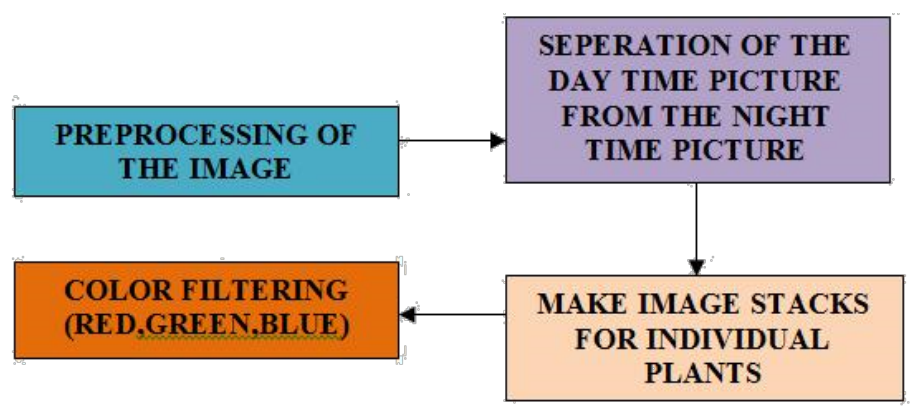

Figure 1 Image Analysis pipeline 
The equations[2] for the linear filtering is shown below.

$\mathrm{D}_{\mathrm{ijk}}=0.35 \times\left(50000-\mathrm{A}_{\mathrm{ik}}\right) / 50000$

$F_{i j k}=\left(G_{i j k}-R_{i j k}+0.4\right) \times\left(1-D_{i j k}\right)+\left(G_{i j k}-R B_{i j k}+0.4\right) \times D_{i j k}$

The image background with value[2] is estimated by:

$\mathrm{T}_{\mathrm{ijk}}=\mathrm{C}_{\mathrm{s}}(\mathrm{s}=$ spring $\mid$ summer $)+0.470 \times \mathrm{U}_{\mathrm{ijk}}-0.00000146 \times \mathrm{A}_{\mathrm{ik}}$

After estimating the image analysis and the threshold of the leaf images the final movement was estimated by rosette detection[2].It is estimated as follows.

$\mathrm{D}_{\mathrm{ik}}=0.35 \times\left(50000-\mathrm{A}_{\mathrm{i}(\mathrm{k}-1)}\right) / 50000$

$\mathrm{F}_{\mathrm{ik}}=\left(\mathrm{G}_{\mathrm{ik}}-\mathrm{R}_{\mathrm{ik}}\right) \times\left(1-\mathrm{D}_{\mathrm{ik}}\right)+\left(\mathrm{G}_{\mathrm{ik}}-\mathrm{RB}_{\mathrm{ik}}\right) \times \mathrm{D}_{\mathrm{ik}}$

$\mathrm{T}_{\mathrm{ik}}=0.12+0.475 \times \mathrm{U}_{\mathrm{ik}}-0.00000130 \times \mathrm{A}_{\mathrm{i}(\mathrm{k}-1)}$

Finally a reduced time of image analysis was estimated in terms of the rosette areas for accession with minimum days.

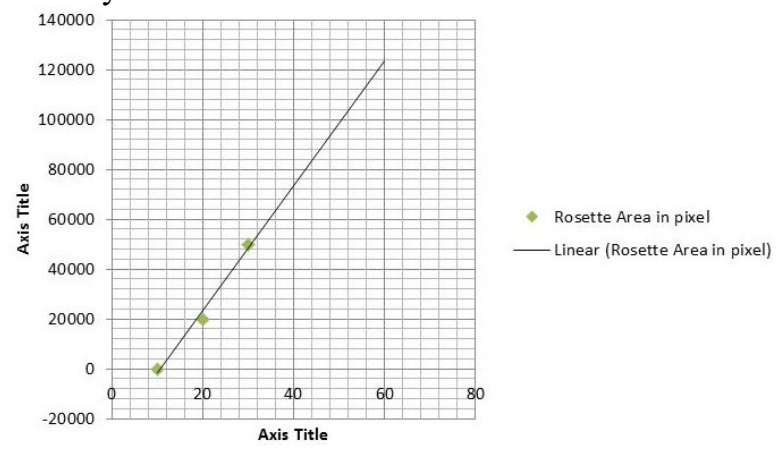

Figure 2 Leaf growth in Spring

The corresponding leaf growth in spring season and summer season are shown in terms of scattered plot diagrams in figure 2 and 3 respectively.

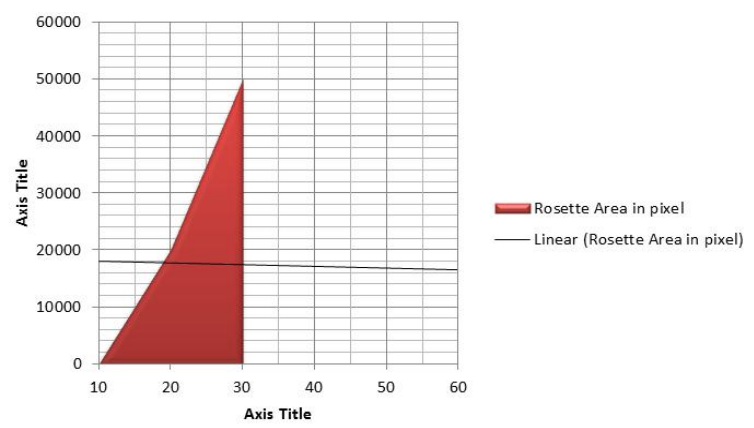

Figure 3 Leaf growing in the summer

The estimated time for different seasons for the growth of leaf was calculated. But this system faced a challenge during the image analysis process since the input images taken were megapixel image and even a small minor change couldn't be recognized.

There was another approach based on leaf segmentation and tracking [3] which used probabilistic parametric active contours. It was an implementation to support agricultural planning. It mainly focuses on measures such as the temperature of the leaves and the 
segmentation of the leaves. In this implementation, a thermal camera was used to periodically monitor the average temperature of the leaves. Probabilistic parametric active contours were used to measure the average rise and fall in the temperature. The results were not optimal in the system implemented because there were noise influences in the contour points. The megapixel images which were captured had many noises in the input image. Hence it was a failure. A multi-modality imagery database for plant phenotyping [5] was proposed. This model aims at producing a potential impact on the plant visual phenotyping. It involved two processes: Capturing of imaging sensors and imaging setup. The imaging sensors involved four types such as fluorescence, infrared, RGB color, and depth. The multi-modality plant imagery database of MSU-PID had a hardware model as shown in figure 4.

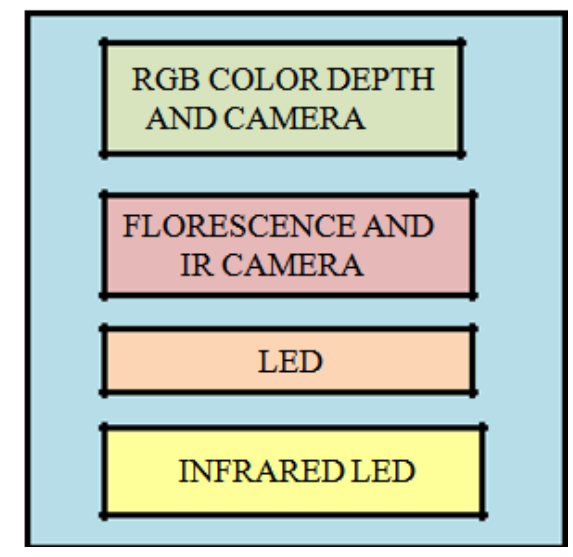

\section{Figure 4 Components of Hardware setup for data collection}

The hardware components were set up as shown above and next a baseline method was carried on to show the multi-leaf segmentation and tracking. Though the method was successful it faced clarity related problems in phenotyping, since the plant crops which covered hectare areas of land couldn't be properly monitored. To overcome the challenges faced by all the approaches[2],[3] and [6] discussed above a new method is proposed using the gigapixel based image analysis. This overcomes the challenge of clarity in the plant images.

\section{Methodology}

The gigapixel input plant image is assumed to have dimensions of $\mathrm{N}$ rows and $\mathrm{M}$ columns. The patches are extracted from the huge gigapixel plant image to a set of patches to extract the set of healthy images and affected plant images. Further, an encoder which is a neural network is used to map the patches[PxPx3] into the embedding vectors. The embeddings are stored in a spatial arrangement keeping the original set of patches. From these original sets of the patches, the features of the plant disease such as height and width are extracted. After the features are extracted the compressed plant image from which the disease needs to be predicted is generated. This compressed image is introduced into a Convolutional Neural Network[CNN] to predict the plant disease in the compressed image from the input gigapixel image. Next, $\mathrm{CNN}$ is trained using the probability estimator algorithm and various plant disease datasets from which the disease needs to be predicted. On arriving at these set of plant diseases 
datasets and the compressed gigapixel images using the Neural Image Compression[NIC] the plant disease is said to be predicted. The architecture of the proposed system is given in the below Figure 5.The proposed gigapixel image analysis for detecting plant disease consists of four modules such as:

1. Image Acquisition and pre-processing of gigapixel images

2. Segmentation and feature extraction

3. Plant disease identification

4. Plant disease prediction using CNN

5. Image Acquisition and pre-processing of gigapixel images

Gigapixel images are very large in size and contains 1000 times the information which is contained in a normal megapixel image. It is quite difficult to acquire the gigapixel images yet the clarity and the information are very effective in it than compared to the normal megapixel images. In this project plant diseases from huge gigapixel images are predicted efficiently using NIC and CNN. The first step is to acquire the gigapixel images.

The input gigapixel plant image taken for analysis in figure 6 which is having a dimension of 0.07 gigapixel. The fetched image tile is shown in the figure 7 which is the input gigapixel image. plant image rendered using the adaptive projection in figure 8 .

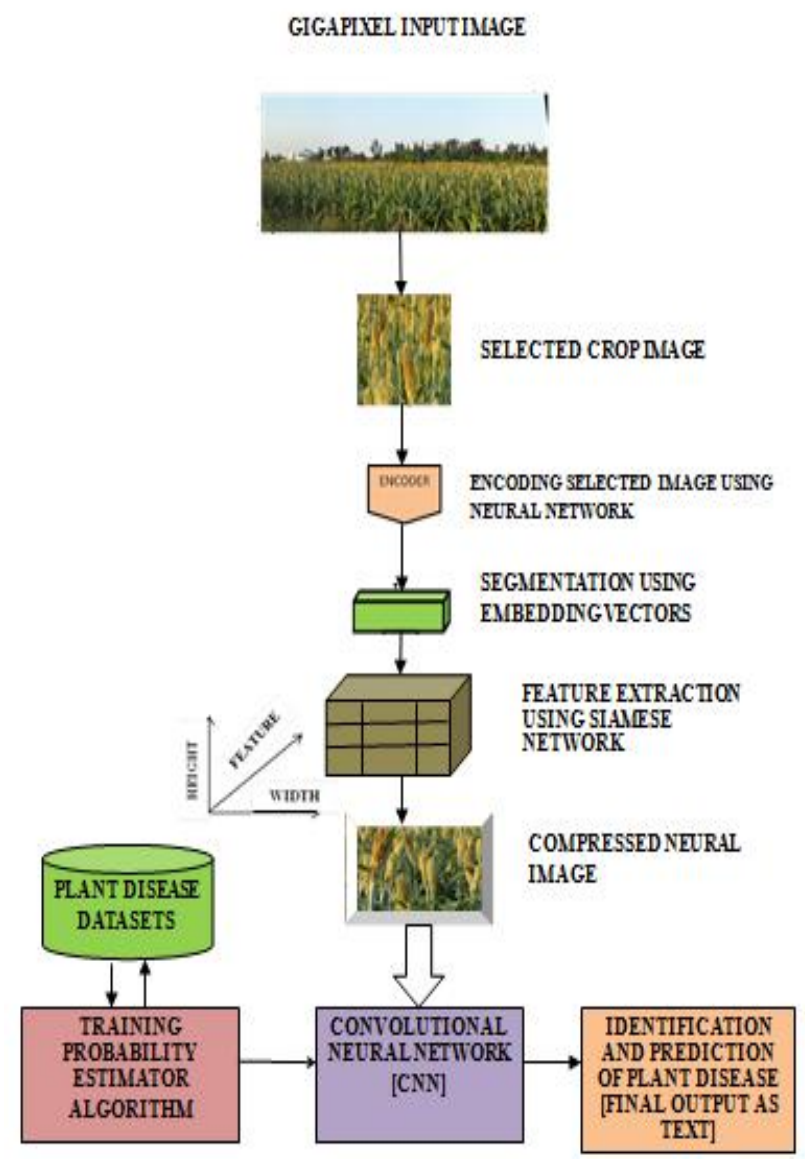

Figure 5 Proposed Gigapixel Image Analysis for detecting disease from a plant 


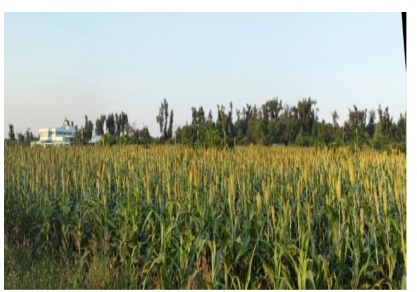

Figure 6 Input Gigapixel

Plant Image(0.07

Gigapixel)

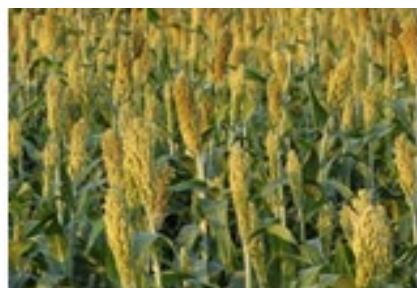

Figure 7 Fetching Image

Tile

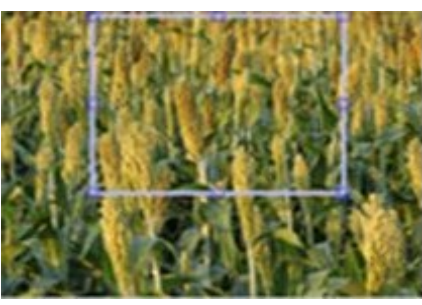

Figure 8 Adaptive projection

\section{Image morphological operations}

\subsection{Preprocessing of image}

The aim of pre-processing is an improvement of the image data that suppresses undesired distortions or enhances some image features relevant for further processing and analysis tasks.In this module, the input image is converted into

-Resized image

-Resized image

- Gray scale Image

-Binary Image

-Unwanted pixels removed Image

The grayscale image is subjected to Gaussian noise.Here Gaussian noise is employed as it produces the best results,since its distribution is greater for values close to zero. The processes involved in the pre-processing are:

1.Image Enchancement

2.Noise Removal

3.Morphologica 1Operation

\subsection{Image Enchancement}

The acquired input gigapixel plant image contains various brown spots in it. To capture the area in which the crop is affected out of a huge crop area found in the gigapixel image, the gigapixel image is first resized and then converted into a grayscale image. The process of converting the input image into a grayscale image for the inputoraize Retiopis shown below in fig 9 which is carried on sequentially. GIGAPXEL MAIZE CROP

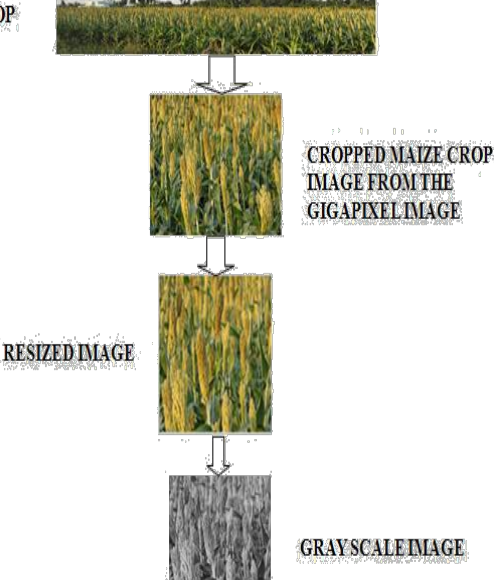

Figure 9 Process of conversion to gray scale image 


\subsection{Noise Removal}

During the process of acquisition of input images due to the computation error in the pixels noises occur. One of the major noise is Gaussian noise. To remove these noises filters are used. Here gigapixel plant images noises are removed using erosion which is a morphological operation. Gaussian noise is given by:

$\mathrm{pG}(\mathrm{z})=\frac{1}{\sigma \sqrt{2 \pi}} e \frac{-(z-\mu)^{2}}{2 \sigma^{2}}$

Where $\mathrm{Z}$ represents Gray Scale level

$\mu$ represents mean value

$\alpha$ represents standard deviation

\subsection{Morphological Operations}

The morphological operations involves two processes:

1.Dilation

2.Erosion

During the process of image enhancement, there is an addition of extra noises by dilation and the noises are removed by erosion. The gray-scale image is converted into a binary image using dilation. The unwanted pixels are removed from the binary image using the erosion process. The above process of image-preprocessing involves the conversion of gigapixel images into the following images:

- $\quad$ Resized image

- Gray scale Image

- $\quad$ Binary Image

- Unwanted pixels removed Image

The pre-processed image is further carried on for the process of segmentation and compressed to a neural image to predict the presence of disease in a gigapixel plant crops using Convolution Neural Network $(\mathrm{CNN})$. The above process of morphological operations involves the process of conversion of the grayscale image which was enhanced into a binary image. The converted binary image of the maize crop in figure 10 .

GRAY SCALE IMAGE

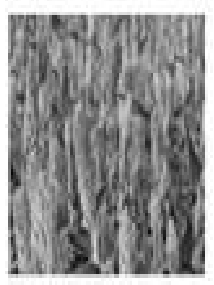

\section{BINARY IMAGE}

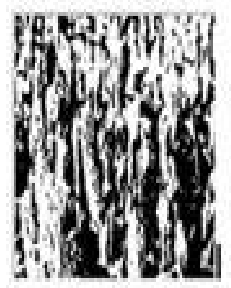

Figure 10 Gray scale image and Binary Image of the input plant Image

\section{Results and Experimentation}

From the pre-processed image of the crop, segmentation is carried on. Segmentation is done using an encoder which splits the pre-processed image into various parts and embeds the segmented portion into a matrix containing various pixels. Feature extraction, detection, and classification of image and text by doing this method we can identify the healthy and disease plant detection process that will be taken place. after all, this process over the $\mathrm{CNN}$ will be trained using a probability estimation algorithm about the chance of plants having the diseases. The convolutional neural networks(CNN) will be trained using the probability estimator algorithm. The Neurally Compressed gigapixel image will be introduced to $\mathrm{CNN}$ and from the trained method the CNN predicts the plant disease. Several encoding strategies, 
namely reconstruction error minimization, contrastive training, and adversarial feature learning are compared and evaluated NIC on a synthetic task of plant disease datasets. The modules included are Acquisition of gigapixel images, Image Pre-processing using morphological operations, Segmentation of gigapixel images, Feature Extraction using probability estimator algorithm, and Detection using Convolutional neural networks(CNN). The plant whose disease must be recognized is pre-processed using matlab as shown below. Figure 11 gives the raw input image. Figure 12 provides the image cropping result. Figure 13 and figure 14 presents in binary and gray scale image respectively. Irreleavant images are removed from the image using figure 15.

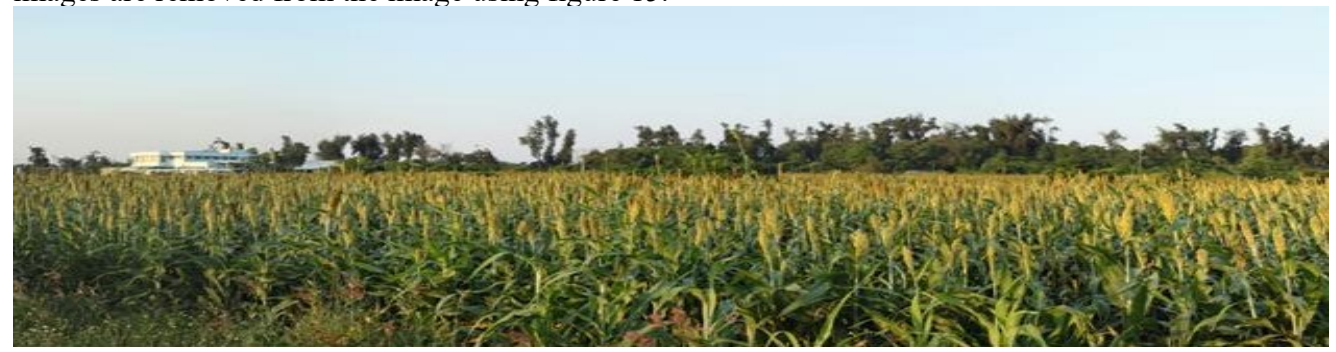

Figure 11 Selected Raw Input Gigapixel Image for analysis

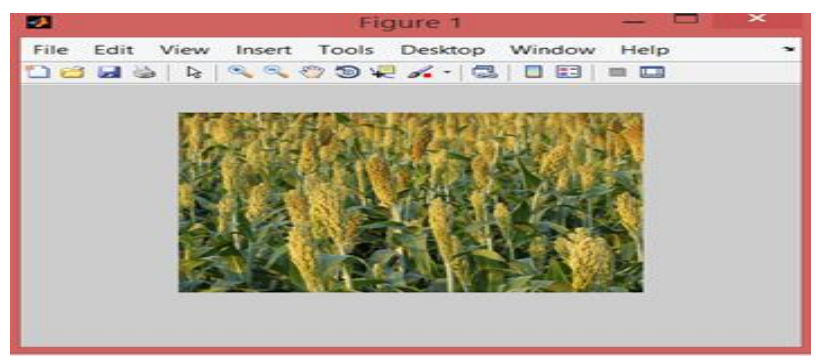

Figure 12 Cropped Image for Pre-processing

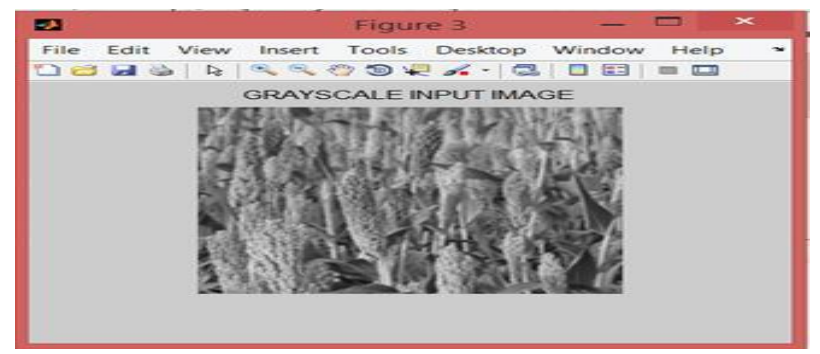

Figure 13 Gray Scale Input Image

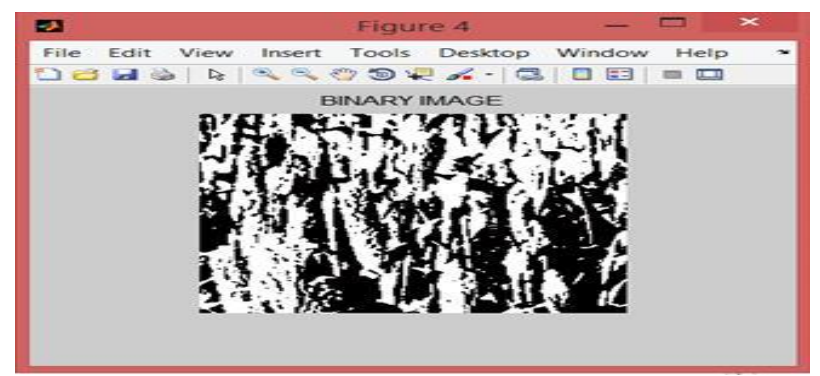

Figure 14 Binary Input Image 


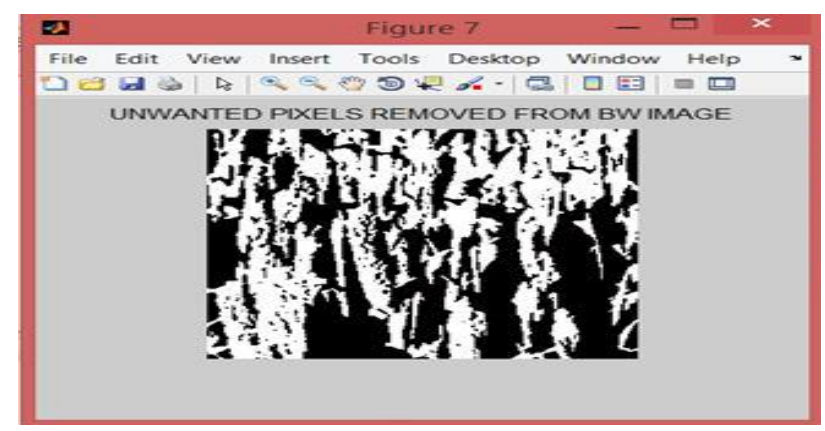

Figure 15 Unwanted Pixels Removal

\section{Conclusion And Future Scope}

Neural Image Compression (NIC) is used for compressing gigapixel plant images and the diseases found in the huge image are predicted by using CNN (Convolutional Neural Networks). The probability estimator algorithm training dataset is based on the universal approximation capability of the original. The overall project concentrates on the rapid development of computer vision and image processing technology; it is possible to improve the accuracy and efficiency of conventional agriculture work such as nutrition deficiency detection and early warning. In this system, we detect various parameters of the plant. The parameters of the leaves are monitored with the help of digital image processing techniques. The controller is used for alerting or indication purposes. This innovative method will be useful to the agricultural fields to monitor the plants and to provide the deficiency of the nutrients at the earliest. This method helped to overcome the issues of the gigapixel image such as time consumption and error-prone process, and limits what machine learning models can learn to the knowledge of human annotators. The main advantage relies on enhanced quality of an image to detect pest, comparatively less in expense, clear visual rating in the image processing method, and no need to develop any aided diagrams for image assessments.

\section{References}

[1] David Tellez, Geert Litjens, Jeroen vander Laak, Francesco Ciompi (2019) _Neural Image Compression for Gigapixel Histopathology Image Analysis ', IEEE Transactions On Pattern Analysis And Machine Intelligence, Vol. X, No. Y, Month 2019.

[2] Xu Zhang, Ronald J. Hause, Jr., and Justin O. Borevitz-Natural Genetic Variation for Growth and Development Revealed by HighThroughput Phenotyping in Arabidopsis thalianall Research School of Biology, The Australian National University, Canberra, Canberra ACT 0200, Australia, 2020

[3] Jeffrey A. Cruz1, Xi Yin2, Xiaoming Liu, Saif M. Imran, Daniel D. Morris, David M. Kramer, Jin Chen, $\|$ Multi-modality imagery database for plant phenotyping,\| Machine Vision and Applications DOI 10.1007/s00138-015-0734-6, 2015

[4] Mario Valerio Giuffrida , Hanno Scharr, Julich, ARIGAN:Synthetic Arabidopsis Plants using Generative Adversarial Network,IEEE International Conference on Computer Vision Workshops, 2017

[5] Jonas De Vylder, Daniel Ochoa, Wilfried Philips, Laury Chaerle,Dominique Van Der Straeten,\|Leaf Segmentation and Tracking Using Probabilistic Parametric Active Contours,\| A. Gagalowicz,W. Philips (Eds.): MIRAGE 2011, LNCS 6930, pp.75-8, 2017 